\title{
Axinos as Cold Dark Matter
}

\author{
Laura Covi
}

\author{
DESY, Notkestrasse 85, D-22603 Hamburg
}

\begin{abstract}
The connection of Dark Matter (DM) to our particle physics model is still one of the open cosmological questions. In these proceedings I will argue that axinos can be successful Cold Dark Matter candidates in models with Supersymmetry and the Peccei-Quinn solution of the strong CP problem. If they are the Lightest Supersymmetric Particle (LSP), they can be produced in the right abundance by thermal scatterings and out of equilibrium decays of the Next-to-Lightest Supersymmetric Particles (NLSPs). Moreover if the NLSPs are charged, their decay could help us understand which is the LSP, e.g. between axino and gravitino.
\end{abstract}

\section{INTRODUCTION}

The nature of Dark Matter is still unknown today, but from simulations of structure formation we know it must be a neutral, Cold, collision-less (i.e. quite weakly interacting) and very long lived particle [1]. Unfortunately such a particle does not exist in the Standard Model (SM): neutrinos are neutral and massive, but so light that they are at most Hot Dark Matter. Therefore we are obliged to look for DM candidates in models beyond the SM. One of the best motivated extensions, supersymmetry with R-parity conservation, naturally gives us a stable massive particle, the LSP. To be DM such a particle has to be neutral and very weakly interacting, so usually only the neutralino or the gravitino are possible LSPs. But if we invoke the Peccei-Quinn solution to the strong CP problem, a new multiplet has to be introduced, the axion multiplet. As long as supersymmetry is unbroken, this whole multiplet remains light, so that no supersymmetric mass parameter is allowed for it (contrary than for the Higgses). After supersymmetry breaking the fermionic component, the axino, obtains a mass, but it still could be the LSP and make a very good DM component. We will present in this talk a summary of axino CDM $\mid 2,3,4,5,6]$ and explore in particular if such particles can be produced in sufficient numbers to make up most of the DM and what that implies for the supersymmetry breaking parameters and collider searches.

\section{PRODUCING AXINOS IN THE EARLY UNIVERSE}

We briefly review here the two main mechanisms that produce axinos in the Early Universe. We concentrate here on the hadronic type of axion models, where is it expected that the axion multiplet does not interact directly with the SM multiplets and therefore the axino does not mix substantially with the standard neutralinos. In the other type of models, this mixing can be larger and the production is therefore enhanced. 


\section{Thermal scatterings}

Any particle, even very weakly coupled, is produced in the thermal plasma by scatterings of the particles that are in thermal equilibrium. Axinos couple directly to the gluons and gluinos due to the axion "anomaly" coupling

$$
W_{P Q}=\frac{g^{2}}{16 \sqrt{2} \pi^{2} f_{a}} \Phi_{a} W^{\alpha} W_{\alpha} \quad \rightarrow \quad \mathscr{L}_{\tilde{a} g \tilde{g}}=\frac{\alpha_{s}}{8 \pi f_{a}} \overline{\tilde{a}} \gamma_{5} \sigma^{\mu v} \tilde{g}^{b} G_{\mu \nu}^{b}
$$

where $\Phi_{a}$ is the axion multiplet containing the axino $\tilde{a}, W$ the gluon vector multiplet containing the gluino $\tilde{g}^{b}$ and the gluon $G_{\mu \nu}^{b}$ and $f_{a}$ is the Peccei-Quinn scale of the order of $10^{11} \mathrm{GeV}$ due to axion physics [7]. So many scattering of the primordial plasma involving colored particles can produce axinos ${ }^{1}$. The axino number density is given by solving a Boltzmann equation of the type

$$
\frac{d n_{\tilde{a}}}{d t}+3 H n_{\tilde{a}}=\sum_{i j}\left\langle\sigma(i+j \rightarrow \tilde{a}+\ldots) v_{r e l}\right\rangle n_{i} n_{j}+\sum_{i}\langle\Gamma(i \rightarrow \tilde{a}+\ldots)\rangle n_{i}
$$

where we are neglecting back-reactions, that are suppressed by $n_{\tilde{a}} \ll n_{i}$.

At high temperature the 2-body scatterings dominate the production, since they contain a vertex given by the dimension 5 operator in eq. (II) and they show a characteristic linear dependence on $T$. So most of the axinos are produced at the highest temperature, the reheat temperature $T_{R}$, and the axino number density is proportional to $T_{R}$. Some of the two body scatterings are IR divergent due to the massless gluon propagator; in the thermal bath such a divergence is screened by the presence of a thermal gluon mass $\simeq g T$. In our computation we introduced such IR cut-off by hand [3]. A self-consistent procedure is instead to perform a full resummation of the Hard Thermal Loops as in [9].

At lower temperatures the decay terms start dominating and the number density is no more proportional to the reheat temperature, it depends instead on the supersymmetric parameters, in particular the gluino and squark masses [4].

Using the expression for the present axino energy density as

$$
m_{\tilde{a}} \frac{n_{\tilde{a}}(T)}{s(T)}=0.72 \mathrm{eV}\left(\frac{\Omega_{\tilde{a}} h^{2}}{0.2}\right)
$$

where $s(T)=2.89 \times 10^{3}\left(\frac{T}{2.726 K}\right) \mathrm{cm}^{-3}$ is the present entropy density, we can then obtain a bound on the reheat temperature as shown in Figure 1.

\section{Out of equilibrium decays}

An axino population is also generated by the NLSP decay after it freezes out from the thermal bath. The heavier superpartners cascade-decay quickly into the NLSP (or

\footnotetext{
1 The same happens also in the case of the gravitino, but with different vertex structure and scale $|8|$.
} 


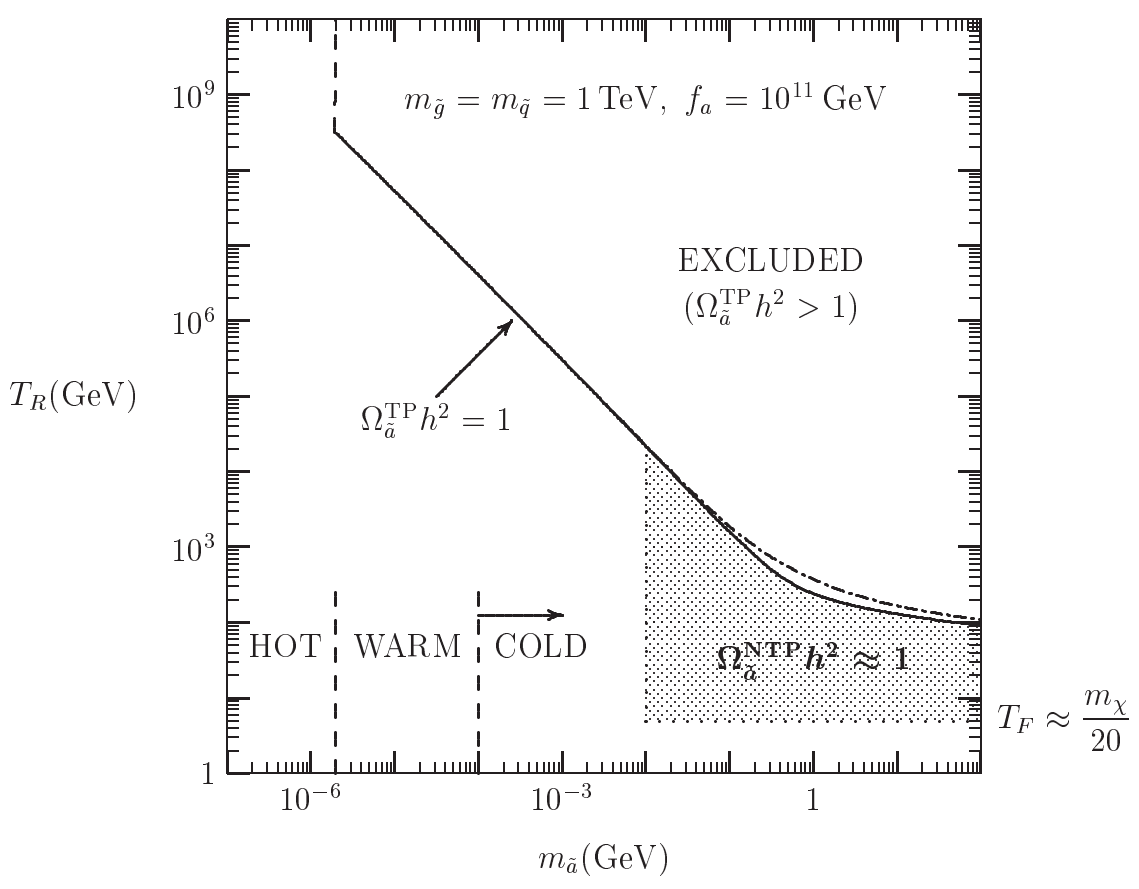

FIGURE 1. Maximal reheat temperature as a function of the axino mass obtained by requiring that the axino energy density is below the present DM density [3]. The difference between solid and dashed line is due to the inclusion of the decay term in the Boltzmann equation ( 2 . In the gray area we expect the non-thermal production via out of equilibrium decays to be also substantial.

very rarely in the LSP as we discussed in the previous section) while still in equilibrium, but the NLSP instead has a lifetime longer than the freeze-out time: in fact all the axino couplings are suppressed by the Peccei-Quinn scale $f_{a} \simeq 10^{11} \mathrm{GeV}$ and so the NLSP lifetime is of the order of seconds or longer. Then the freeze-out process is unaffected and the decay takes place only much later as shown in Figure 2.

In this case, the axino number density can be directly computed from the NLSP would-be-relic number density as

$$
\Omega_{\tilde{a}}^{N T}=\frac{m_{\tilde{a}}}{m_{N L S P}} \Omega_{N L S P}
$$

If the mass ratio is not too small, we still have a connection with the classical WIMP mechanism in case the NLSP is a neutralino or a stau.

On the other hand, a couple of problems can arise if the decay happens too late:

- Big Bang Nucleosynthesis can be spoiled by the energetic "active" particles produced in the decay along with the axino: the strong limits on the injection of energetic particles depend on the electromagnetic/hadronic nature of the produced showers and the decay time [10]. In general such limits are weak for the axino case since the NLSP lifetime (excluding a strong mass degeneracy) is below $10^{2} s$, but they can affect the region of small mass for both the neutralino and stau NLSP $[3,4 \mid$. 


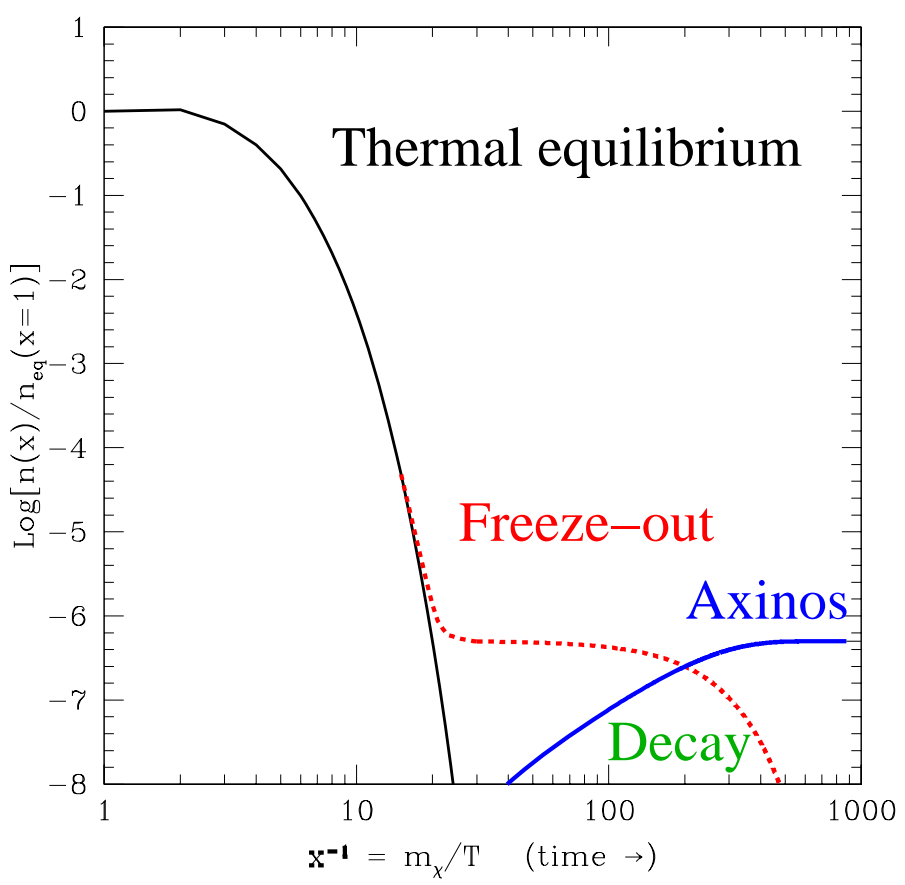

FIGURE 2. Freeze-out of the NLSP and subsequent decay into axino. Due to R-parity conservation the number of axino produced in the decay is the same as the NLSP number.

- Are axino from the decay cold enough to be CDM ? They are relativistic at production even if the NLSP is not and have a non-thermal spectrum:

$$
v(T)=\frac{p(T)}{m_{\tilde{a}}} \simeq \frac{m_{N L S P}}{2 m_{\tilde{a}}}\left(\frac{g_{*}(T)}{g_{*}\left(T_{d e c}\right)}\right)^{1 / 3} \frac{T}{T_{d e c}},
$$

where $T_{d e c}$ is the temperature at the decay time. So the question is if they have sufficient time to cool down before structure formation begins. In [11] such constraints have been studied and the conclusions is that an axino mass at least of order of $1 \mathrm{GeV}$ is probably needed.

\section{AXINOS AND THE CMSSM}

Depending on the parameters and $T_{R}$, either production mechanism can dominate and produce sufficient axinos to explain the present DM density. In general either $T_{R}$ is bounded as in Fig. 1 or the axino is so light to be a subdominant (warm or hot) DM component. In the last case in our scenario the axion [7] could be the DM.

Assuming that the axinos are $\mathrm{CDM}$ and that the supersymmetric partners of the SM particles can be described by the Constrained MSSM, we can see which is the preferred parameter region depending on the production mechanism. In the CMSSM the superparticle spectrum and couplings are simply function of the SM and additional four parameters: the ratio of the Higgs v.e.v. $\tan \beta$, the gaugino and scalar masses $m_{1 / 2}, m_{0}$ 


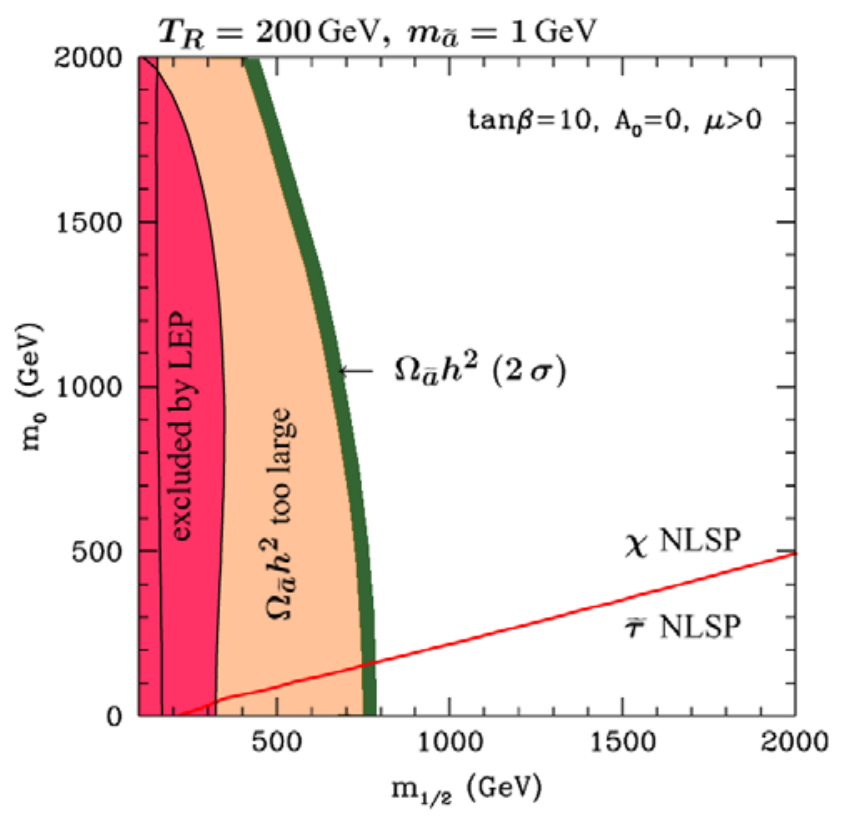

FIGURE 3. Allowed parameter space for the case of dominant thermal production [5]. We have chosen here $T_{R}=200 \mathrm{GeV}, m_{\tilde{a}}=1 \mathrm{GeV}$ and $f_{a}=10^{11} \mathrm{GeV}$. The dark gray strip gives axino in the right abundance to explain all DM, while the lighter gray areas are excluded by LEP constraints or too large axino number density. The white area has too low axino density to explain DM.

and the trilinear coupling $A_{0}$, which are universal at the GUT scale. The modulus of the $\mu$ parameter is fixed by radiative electroweak symmetry breaking and we will always consider the positive sign in the following.

\section{Mostly thermal production}

In the case of high $T_{R H}$ all the particles in the thermal bath can be treated as massless and so there is practically no dependence on the supersymmetry breaking parameters. On the other hand if we require the axino mass to be above $1 \mathrm{GeV}$, the reheat temperature has to be sufficiently low and comparable to the superparter masses, so the decay term in the Boltzmann equation become important and a strong dependence on the gluino mass appears also due to the squark-quark-axino coupling [4]. We have then that the allowed region is a narrow band in the gaugino mass parameter with a much smaller dependence on $m_{0}$ as shown in Figure 3. Note that in this case the small gaugino mass region is excluded because there too many axinos are produced. The exact position of the allowed band though strongly depends on the chosen reheat temperature and it moves at larger $m_{1 / 2}$ for larger $T_{R}$. 


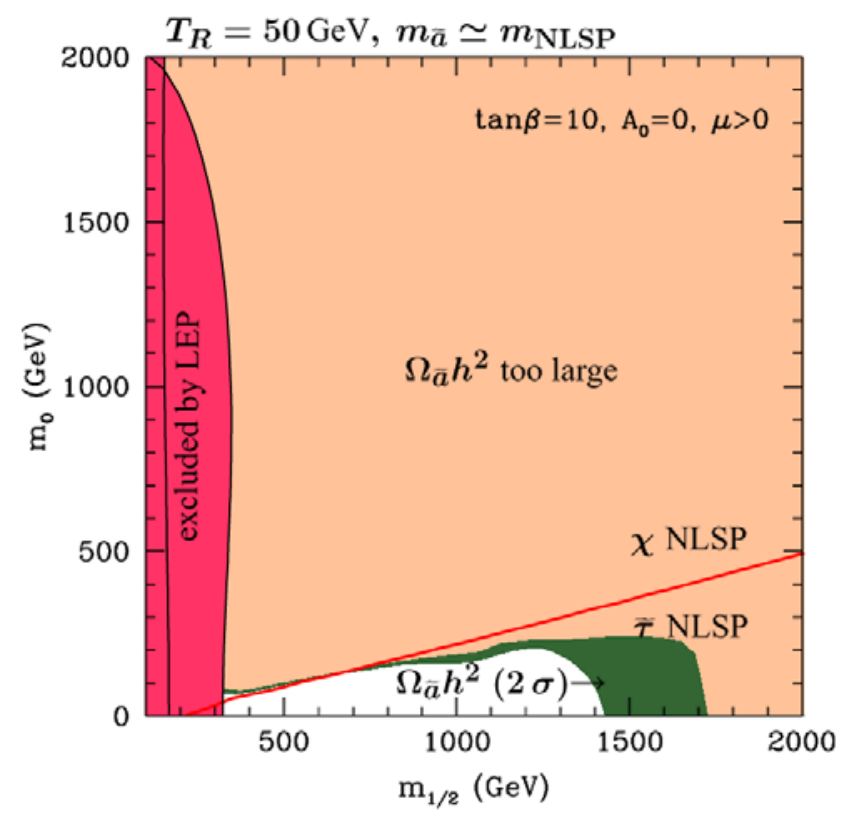

FIGURE 4. Allowed parameter space for the case of dominant production via out of equilibrium NLSP decay 5]. We have chosen here $T_{R}=200 \mathrm{GeV}, m_{\tilde{a}}=1 \mathrm{GeV}$ and $f_{a}=10^{11} \mathrm{GeV}$. The dark gray region gives axino in the right abundance to explain all DM, while the lighter gray areas are excluded by LEP constraints or too large axino number density. The white area has too low axino density to explain DM.

\section{Mostly out of equilibrium NLSP decay}

In the CMSSM the NLSP can be either the neutralino or the stau. The latter happens in the wedge with low $m_{0}$, that is usually considered excluded if the stau is the LSP. In our case though the LSP is the axino and even the stau wedge is viable; in particular there is a wide region where the stau decay can produce the right amount of axino DM as we see in Fig. 4. On the other hand there is also a tiny strip in the neutralino NLSP region, analogous to the neutralino DM region, but shifted slightly due to the rescaling in eq. 4. Both regions are practically unaffected by BBN constraints contrary to what happens for the gravitino case [12].

\section{HOW TO DISTINGUISH THE LSP ?}

If the axino is the LSP, very different signals could be found at colliders depending on the nature of the NLSP. If the neutralino is the NLSP, the only way to find out that it is not DM is if the mass and cross sections turn out to give a too large neutralino number density or to be excluded by direct DM searches. Then we would have good reasons to say that the neutralino must be unstable, but to study its decay will be very difficult.

If the stau (or another charged sparticle) is the NLSP instead, we will have the striking signal of an apparently stable charged heavy particle in the detector. In that case it will 
be clear that the LSP must be a very weakly interacting particle, but to know which one, we will need to measure and study the NLSP decay. In particular to distinguish between axino and gravitino, that can give similar NLSP lifetimes, we will need to measure the branching ratio and the angular dependence of the radiative decay in order to reach a definitive identification [6].

\section{CONCLUSIONS}

Axinos with masses in the $\mathrm{MeV}-\mathrm{GeV}$ range are good $\mathrm{CDM}$ candidates for low reheat temperature: they can be produced either from thermal processes or from NLSP decay with the right abundance. Such scenario is analogous to the gravitino LSP one, but an axino LSP evades more easily BBN bounds, since the NLSP lifetime is shorter than $10^{2} \mathrm{~s}$. Therefore both neutralino and stau NLSPs are allowed in our case.

Compared to neutralino DM, different regions of the CMSSM parameter space become allowed and preferred, in particular even heavier sparticles masses and a charged NLSP like the stau. An apparently stable charged particle would surely give a striking signal at the LHC and ILC and indicate that the neutralino is not the DM. On the other hand, disentangling between LSP candidates will require to stop such NLSPs and measure their decay; in particular axino and gravitino will be distinguished if a sufficient number of radiative decay can be observed.

\section{ACKNOWLEDGMENTS}

It is a pleasure to thank A. Brandenburg, K. Hamaguchi, H.B. Kim, J.E. Kim, R. Ruiz de Austri, M. Small, F.D. Steffen and in particular L. Roszkowski for several years of fruitful and exciting collaboration. The author would also like to thank the Organizers for the exciting Workshop and their patience in waiting for these proceedings. The author acknowledges the European Network of Theoretical Astroparticle Physics ILIAS/N6 under contract number RII3-CT-2004-506222 for financial support.

\section{REFERENCES}

1. G. Bertone, D. Hooper and J. Silk, Phys. Rept. 405 (2005) 279 [arXiv: hep-ph/0404175 .

2. L. Covi, J. E. Kim and L. Roszkowski, Phys. Rev. Lett. 82 (1999) 4180 [arXiv hep-ph/9905212 .

3. L. Covi, H. B. Kim, J. E. Kim and L. Roszkowski, JHEP 0105 (2001) 033 [arXiv hep-ph/0101009.

4. L. Covi, L. Roszkowski and M. Small, JHEP 0207 (2002) 023 [arXiv: hep-ph/0206119 .

5. L. Covi, L. Roszkowski, R. Ruiz de Austri and M. Small, JHEP 0406 (2004) 003 [arXiv hep-ph/0402240 .

6. A. Brandenburg, L. Covi, K. Hamaguchi, L. Roszkowski and F. D. Steffen, Phys. Lett. B 617 (2005) 99 [arXiv hep-ph/0501287.

7. See the contributions of B. BeltrÃąn and E. Masso to these proceedings.

8. M. Bolz, A. Brandenburg and W. Buchmuller, Nucl. Phys. B 606 (2001) 518 [arXiv: hep-ph/0012052

9. A. Brandenburg and F. D. Steffen, JCAP 0408 (2004) 008 [arXiv: hep-ph/0405158 .

10. M. Kawasaki, K. Kohri and T. Moroi, Phys. Rev. D 71 (2005) 083502 [arXiv astro-ph/0408426.

11. K. Jedamzik, M. Lemoine and G. Moultaka, JCAP 0607 (2006) 010 [arXiv astro-ph/0508141 . 
12. See the contribution of K.Y. Choi to these proceedings; also F. D. Steffen, arXiv: hep-ph/0605306 and references therein. 